\title{
High Sensitivity All-fibre Methane Sensor with Gas Permeable Teflon/Cryptophane-A Membrane
}

\author{
Rand Ismaeel1,2, Alexander Beaton ${ }^{2}$, Andrei Donko', Wanvisa Talataisong1, Timothy Lee ${ }^{1}$, Thierry \\ Brotin $^{3}$, Martynas Beresna1, Matthew Mowlem² and Gilberto Brambilla1 \\ 1. Optoelectronics Research Centre, University of Southampton, Southampton SO17 1BJ, U.K. \\ 2. Ocean Technology and Engineering Group, National Oceanography Center, Southampton SO14 3ZH U.K. \\ 3. Laboratoire de chimie, Univ. Lyon, Ecole Normale Supérieure de Lyon, CNRS UMR 5182, Université Lyon 1, 46 Allée d'Italie \\ Lyon F69364, France.
}

We demonstrate a simple, cheap and compact all fibre methane sensor, with record sensitivity of $0.16 \mathrm{~nm} / \mathrm{ppm}$, composed of a D-shaped cross section optical fibre in between two fibre Bragg gratings. The flat side of the fibre is coated with a unique membrane used to solely detect methane, made by a gas permeable Teflon layer and doped with cryptophane-A molecules.

Methane is a greenhouse gas, which is 25 times more potent than carbon dioxide in its capacity to trap heat in the Earth's atmosphere [1]. Current technologies available for methane detection (e.g. [2,3]) are still orders of magnitudes lower than those required for studying climate change. Optical sensors offer all the advantages of miniaturization, high sensitivities and detection limits; however, their sensitivity typically scales proportionally with fibre length, which is directly related to instrument size leading to bulky devices.

In this work, we present a compact, miniaturized high sensitivity sensor, based on a D-shaped fibre (Phoenix Photonics Ltd). The polished side of the fibre was coated with a Teflon membrane enriched with cryptophane molecules. Cryptophanes are host molecules that can bind reversibly with a variety of guest molecules [4], with Cryptophane-A showing strong affinity towards methane. The thin membrane was prepared by dissolving $2 \mathrm{mg}$ of cryptophane-A molecules in acetone, which was then mixed with $5 \mathrm{~g}$ of Teflon-AF. The flat side of the D-shaped fibre allows a strong evanescent field extending into ambience and thus interacting with the membrane. Meanwhile, the grating cavity realizes a long effective interaction path length through the resonance effect, while using a short physical fibre length $(1 \mathrm{~cm})$. The cavity was fabricated by using femtosecond direct write to inscribe Bragg grating reflectors at both ends of the fibre.

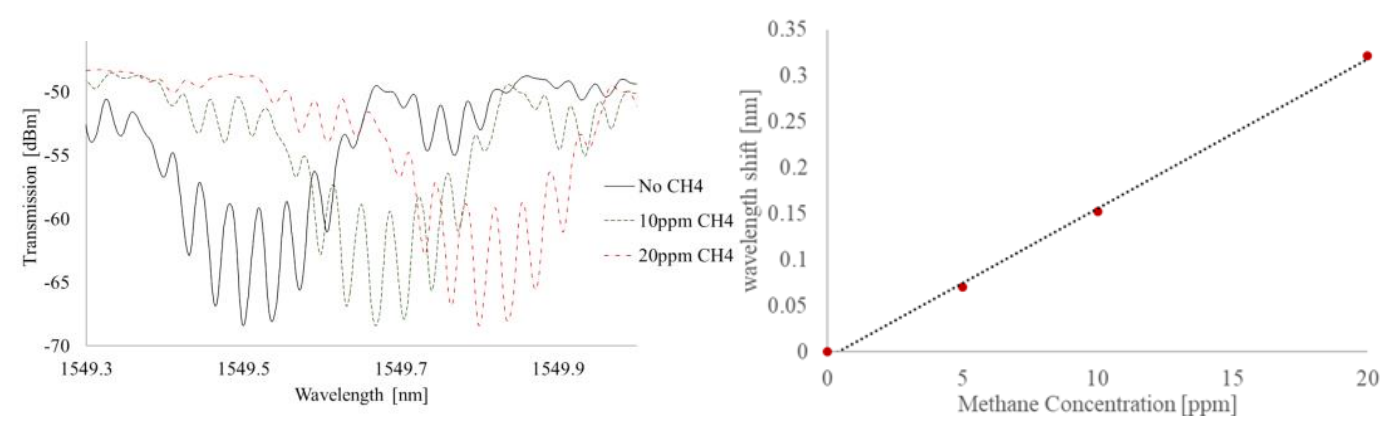

Fig. 1 (a). The transmission spectrum change related to changing the refractive index of methane by varying concentration. (b) The linear relationship between the wavelength shifts versus the methane concentration.

The all fibre sensor was placed inside a custom made flow cell, which was connected to a vacuum chamber. Gas cylinders of known concentrations of methane were connected to the cell inlet. The input and the output of the fibre sensor were connected to an incoherent white light source and an optical spectrum analyser, respectively. In this experiment, three different concentrations of methane were recorded: 5, 10 and 20 ppm. Fig.1 (a, b) shows changes in the optical spectrum associated with varying the methane concentration. A clear linear spectrum shift response to different concentrations was observed in the fibre output. A record sensitivity of $0.16 \mathrm{~nm} / \mathrm{ppm}$ was obtained from this simple and low cost sensor with a response time of $4 \mathrm{~s}$. Optimising the thickness and the concentration of cryptophane in the membrane is predicted to double the sensitivity of the device and enhance the response time considerably. Initial results also showed that 2 orders of magnitude sensitivity enhancement could be obtained by replacing the D-fibre in the same configuration with an optical microfibre with a waist diameter of $\sim 2 \mu \mathrm{m}$. This would improve the proposed sensor, making it capable of sensitivities comparable to single molecule detection.

\section{References}

[1] Yvon-Durocher, G. et al. Methane fluxes show consistent temperature dependence across microbial to ecosystem scales. Nature 507, 488-491 (2014)

[2] G. Williams, G.S.V. Coles, “The semistor: a new concept in selective methane detection," Sens. Actuators B 57, 108-114 (1999).

[3] O. Hennig, R. Strzoda, E. M'agori, E. Chemisky, C. Tump, M. Fleischer, H. Meixner, I. Eisele, "Hand-held unit for simultaneous detection of methane and ethane based on NIR-absorption spectroscopy," Sens. Actuators B 95, 151-156 (2003).

[4] T. Brotin, J.-P. Dutasta, Chem. Rev., 88-130 (2009). 